\title{
Quality of Life in Patients With Liver Cirrhosis: A Systematic Review
}

\author{
Marzieh Pazokian' ${ }^{1}$ Maryam Esmaeili2 ${ }^{{ }^{*}}$ \\ ${ }^{1}$ School of Nursing and Midwifery, Skull Base Research Center, Loghman Hakim Hospital, Shahid Beheshti University \\ of Medical Sciences, Tehran, Iran \\ ${ }^{2}$ Student Research Center, Faculty of Nursing and Midwifery, Shahid Beheshti University of Medical Sciences, Tehran, \\ Iran
}

*Corresponding Author: Maryam Esmaeili, MSc Student of Nursing, Student Research Center, Faculty of Nursing and Midwifery, Shahid Beheshti University of Medical Sciences, Tehran, Iran. Tel: +98-9122267194,

Email: m.esmaeili841@gmail.com

Received April 30, 2019; Accepted July 2, 2019; Online Published November 5, 2019

\begin{abstract}
Background: Quality of life (QOL) is a powerful phrase that reflects maintaining health and well-being in different societies and cultures, reflecting the positive and negative aspects of people's lives. Patients with liver cirrhosis (LC) have an undesirable QOL, and this has become one of the most important causes of mortality in the world.

Objective: This review article aimed to summarize the results of previous studies on health-related QOL in patients with LC to determine their problems and needs.

Methods: This review was conducted using Cochran's seven-step model. This paper is the result of research on QOL in patients with LC through searches conducted in Google Scholar, PubMed, and Science Direct using the keywords liver cirrhosis, chronic liver disease, quality of life, health-related quality of life, and self-care for articles published between 2012-2018. Ultimately, 11 articles were obtained and analyzed.

Results: The results of this review showed that the QOL in patients with LC is poor and is lower than in other populations. Many factors, such as age, gender, and education, influence QOL and should be addressed in order to solve the challenges and problems faced by these patients. Increasing awareness and education are the most important issues for LC patients.

Conclusion: QOL in patients with LC is affected by many factors. The lack of awareness about the disease and its complications is considered the biggest problem for these patients. To improve the QOL and care of these patients, nursing care guidelines, awareness raising, and education about the disease and its complications, treatment, and new treatments are needed.

Keywords: Cirrhosis, Quality of Life, Self-care, Chronic Liver Disease
\end{abstract}

\section{Background}

Chronic liver disease (CLD) is one of the most common diseases in the world. In Korea, CLD is one of the most prevalent diseases. It is linked to diabetes and hypertension and causes $13.3 \%$ of all deaths; it was ranked the eighth cause of death in 2016. ${ }^{1,2}$ Liver disease is often sub-clinical, and has led to an increase in liver cirrhosis (LC) cases in many countries, especially developing ones. ${ }^{3}$ Because symptoms are not obvious at disease onset, precise statistics for this disease in Iran are not available. ${ }^{4}$ In the United States, 60000 deaths occur annually due to LC. Furthermore, the hospitalization time of these patients has increased. ${ }^{3}$ In the United Kingdom, at least 7000 new LC cases are detected every year. ${ }^{4}$

The number of people with LC will continue to increase because of the prevalence of hepatitis $\mathrm{C}$ among people during the 1950s-1960s. ${ }^{5}$ LC is a chronic and irreversible liver disease that affects a large number of organs and systems in the body and reduces the quality of life (QOL) of patients. ${ }^{1,5} \mathrm{LC}$ is the result of a liver tissue replacement with fibrosis, oscillation, or nodular regeneration that progresses to the loss of liver function. When the disease progresses, the only way to treat it is with liver transplantation. ${ }^{4}$

Initially, many patients with LC show non-specific symptoms that do not directly indicate liver disease. These symptoms include lethargy, fatigue, numbness, and nausea. As the disease progresses to portal vein hypertension, signs and symptoms appear in patients. ${ }^{5}$ Analysis of lifestyledependent factors in patients with LC has shown that alcohol consumption, smoking, obesity (BMI increasing), and the level of education of patients are significant. ${ }^{6}$ These factors have different impacts in different geographical regions. For example, in Iran, hepatitis B is the leading cause of hepatic cirrhosis, while alcohol in the United States and European countries are main causes. ${ }^{3}$

Patients with LC suffer from symptoms such as fatigue, joint pain, itching, appetite loss, depression, anxiety, weakened libido, memory problems, protein energy malnutrition, ${ }^{1,7,8}$ jaundice, increased bleeding, abdominal distension, ascites, peripheral edema, dyspnea, mental

Copyright (C) 2019 The Author(s). This is an open-access article distributed under the terms of the Creative Commons Attribution License (http:// creativecommons.org/licenses/by/4.0), which permits unrestricted use, distribution, and reproduction in any medium, provided the original work is properly cited. 
changes, emotional problems, ${ }^{3,9}$ weight loss, muscle cramps, and the inability to work. ${ }^{5}$

These symptoms are associated with reduced QOL and psychological distress in LC patients and disrupt patients' daily life and activities. ${ }^{3}$ Obviously, LC patients' needs for healthcare are substantial. Contrary to other diseases, such as heart failure or chronic obstructive pulmonary disease, models of community supportive and home care have not been developed for LC patients. ${ }^{5}$

The occurrence of physical problems affects the function and efficiency of LC patients and decreases their satisfaction with life. ${ }^{1,8}$ People with LC often suffer from malnutrition, loss of energy, and protein-energy malnutrition (PEM). PEM is one of the most important side effects of LC that greatly reduces the physical activity of patients. In most cases, sarcopenia occurs, which reduces skeletal muscle and increases muscle weakness. ${ }^{9}$

Life is accompanied by many problems for LC patients, and their QOL is decreased dramatically. ${ }^{1}$ QOL has a high correlation with the rate of illness and mortality in these patients. Patients with LC need to follow a list of dietary and drug restrictions and have regular laboratory tests and frequent follow-ups. Because of the important role nutrition plays in the prognosis of cirrhotic patients, nutritional studies on LC patients should be done. Caregivers should be mindful of the patient's medical history, physical exam, duration of illness, and biochemical information. Laboratory values that determine nutritional status are albumin, pre-albumin, prothrombin time, creatinine, and, indirectly, immune system function. ${ }^{7}$ The prognosis of LC can be improved through the development of effective interventions, including control of gastrointestinal bleeding, hepato-renal syndrome, spontaneous bacterial peritonitis, liver cancer, and hepatic encephalopathy. Health-related quality of life (HRQOL) is very important in cirrhotic patients, and it is affected by the many physical and psychological challenges that LC patients face. It should be noted that these problems and challenges may not be evaluated using general tools. Therefore, it is necessary to provide a specific questionnaire for LC patients. Diagnosing factors related to LC may help provide special care for them and guidance for future research. ${ }^{7,10}$ It can also provide necessary strategies to promote new therapies and help healthcare providers. ${ }^{11}$ This issue is more important in the long-term care of outpatients. ${ }^{8}$

\section{Objective}

Despite the importance of LC and its increasing prevalence, there are no studies concerning cirrhotic patients' needs and problems or strategies for solving cirrhotic patients' issues. Assessing the HRQOL of LC patients can lead to the identification of their problems and needs. It will provide the conditions for developing a comprehensive care plan for patients. In many cases, it will also reduce the challenges that patients face, improve their life expectancy, and increase their survival rates.

\section{Methods}

The purpose of this review was to determine the QOL of patients with LC and to determine the problems and needs of these patients. Articles published between 2012 and 2018 were identified by searching Pub-Med, Ovid, Google Scholar, and Science Direct databases using the keywords QOL, health related quality of life (HRQOL), chronic liver disease (CLD). LC was initially selected as the main subject, and then HRQOL and problems and needs of patients with LC were added. The second step was to find and identify the papers most relevant to this study. Papers published between 2012 and 2018 were chosen for this study, and Cochran's seven-step model was used to perform the review as follows:

Step 1: Specifying the year.

Step 2: Determining inclusion criteria, which were articles published in English and Persian, quantitative and qualitative studies, and availability of full text. In this step, 120 papers were selected.

Stage 3: Study selection. LC was selected as the main subject. Then, the subjects of health-related QOL and problems and needs were added. After an evaluation of the titles and summaries, 80 articles which were more related to the main title were selected and the other papers were excluded. The steps for selecting articles are summarized in Figure 1.

Step 4: Assessment of study quality. In this step, the full text of the papers was studied. In all, 25 papers with important content about QOL in LC patients were selected and analyzed.

Step 5: Data extraction. After studying the full text of the papers, 11 papers that had important QOL points in patients with LC are selected.

Step 6: Data analysis. Data from the review of the studies are presented in Table 1.

Step 7: Presentation of results. The results of this review are presented as research findings.

\section{Results}

The results show that patients with LC suffer low HRQOL, which can increase the mortality rate among them. Many factors, such as age, gender, anxiety, literacy level, depression, economic problems, and physical complications affect HRQOL in LC patients. Most patients know very little about their disease. The two most important factors in patients with LC are increased awareness and attention to their educational needs. To achieve higher efficacy, steps to increase education and attention should be taken in the early stages of the disease. Educational and nursing interventions as well as drug therapies are considered the key to success in LC patients and will increase the patients' level of awareness, HRQOL, education, life expectancy, and survival rate.

\section{Discussion}

The current study analyzed QOL in patients with LC to determine their problems and needs. All studies introduce 


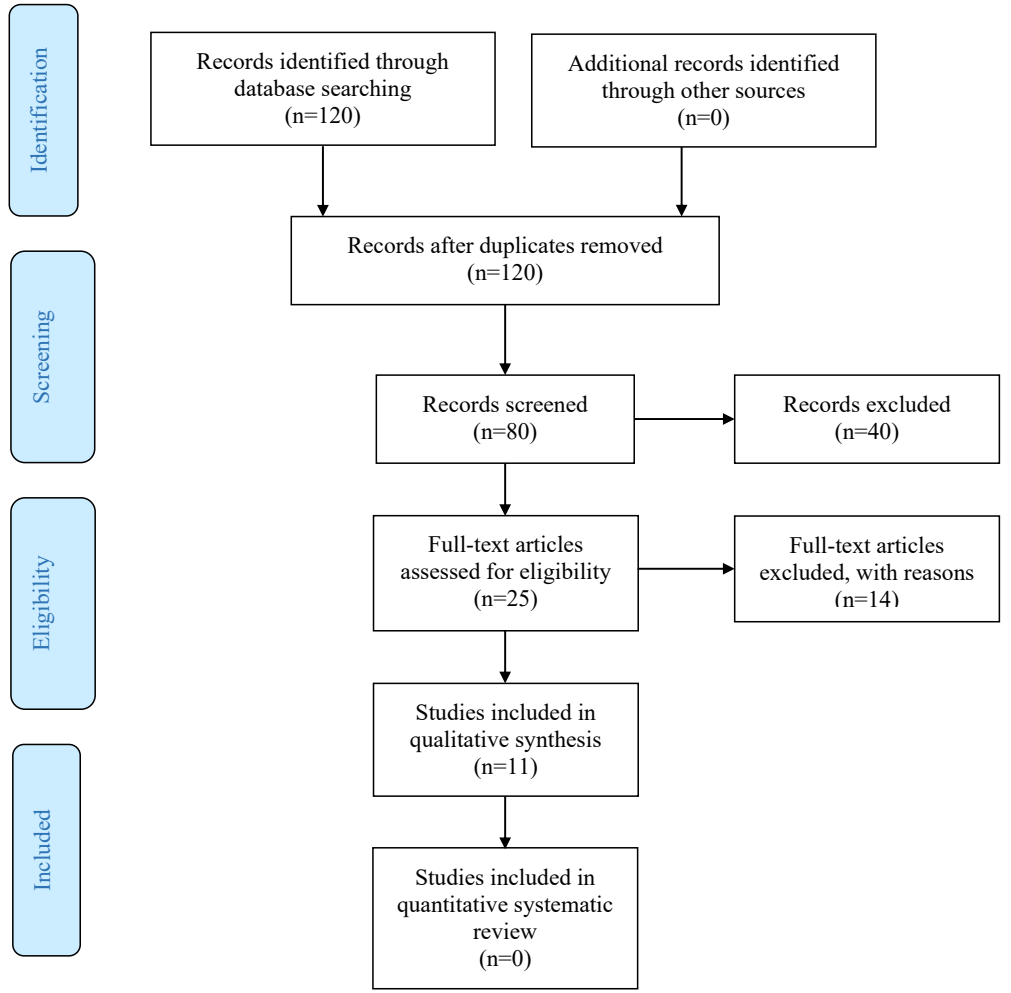

Figure 1. The PRISMA Flow Diagram of Reviewing the Articles for QOL of Patients With Cirrhosis of the Liver.

LC as a growing and irreversible disorder of the form and function of liver cells. LC is life threatening and can create many challenges for patients. ${ }^{11,12} \mathrm{HRQOL}$ is one of the most important and necessary factors in evaluating patients' cure, and it can predict life expectancy. Therefore, it must be considered in patients with chronic disease. ${ }^{12}$ Various factors affect the QOL of LC patients, including physical conditions and demographic characteristics. The problems and challenges facing patients with LC can be seen in the physical, psychological, social, and economic parts of their lives and in their families. These problems affect not only the patients' QOL, but also their treatment process. The needs of LC patients in the two areas of knowledge and education are remarkable. Studies have shown that patients' have little knowledge about the disease, treatment process, new treatments, and complications. Despite the importance of HRQOL, it has not been well evaluated in patients with CLD compared to other diseases. Factors in nursing care, which affect the QOL of these patients, have especially been ignored. ${ }^{1,13}$

\subsection{Factors affection QOL in LC Patients}

HRQOL can be reduced by impaired physical function and reduced patient capacity. Since cirrhosis has many effects on patients, other family members, health care providers, and society, it is necessary to identify the factors that are important in this field. Understanding the factors affecting HRQOL depends on understanding the multiple factors of LC and an individual's health status. ${ }^{5}$ Although there is limited information about the association between psychological symptoms and QOL in patients with LC, the relationship has been confirmed by Nardelli et al, ${ }^{21}$ who stated that depression, anxiety, and alexithymia had reversible relationships with the QOL of LC patients. Alexithymia was reported in $25 \%$ of people with LC, while its rate was noted as being between 5-17\% among healthy people in the same age group. ${ }^{12}$

Loria et al detected many factors that can affect HRQOL and the results of treatment in patients with LC, including general health, social support, and balance in care. The expansion of cirrhosis, reduction in liver function, ${ }^{1,5}$ and the limitations and side effects of the illness ${ }^{14}$ are very important. Other effective factors include demographic information such as age, gender, marital status, level of education, and economic situation. ${ }^{1,15,16}$ These factors have different statistical impacts in different societies. ${ }^{1,17}$ For example, economic situation, gender, and age have greater effects on HRQOL in Germany, while age and marital status are more effective in China. In fact, being married and having familial support have positive effects on the HRQOL of LC patients. ${ }^{1}$

Nardelli et al confirmed that depression, anxiety, and alexithymia have inverse relationships with QOL. Alexithymia affects $25 \%$ of LC patients, while $5 \%-17 \%$ of healthy people of a working age suffer from it. ${ }^{18}$ Other effective factors are spread of disease (44\%) and economic problems related to treatment and prescribed medications (22\%). Behavioral factors such as alcohol consumption and lack of sleep have important effects on HRQOL as well. According to previous studies, sleeping less than 6 hours 
Table 1. Results of Research on the QOL of Patients With Cirrhosis of the Liver

\begin{tabular}{|c|c|c|c|c|}
\hline Author & Year & Country & Sampling & Results \\
\hline Kim et $\mathrm{al}^{1}$ & 2018 & Korea & 134 (30-70 years) & $\begin{array}{l}\text { In this study, } 85.6 \% \text { of patients reported morbidity. There were also significant differences between } \\
\text { age, gender, education level, and marital status and HRQOL. Marriage and marital support had } \\
\text { positive effects on liver function and HRQOL in patients. The lowest HRQOL was seen in patients } \\
\text { with hepatitis C. }\end{array}$ \\
\hline $\begin{array}{l}\text { Fagerström } \\
\text { et } \mathrm{al}^{7}\end{array}$ & 2017 & $\begin{array}{l}\text { South } \\
\text { Sweden }\end{array}$ & 13 (46-70 years) & $\begin{array}{l}\text { In this study, patients described how they experienced their life situation and how they managed their } \\
\text { lives. They reported many problems such as fatigue, bad sleep, reduced activities, emotional distress, } \\
\text { leg edema, and weight. }\end{array}$ \\
\hline $\begin{array}{l}\text { Hamzetaha } \\
\text { et } \mathrm{al}^{2}\end{array}$ & 2015 & Minia & 50 (20-60 years) & $\begin{array}{l}\text { This study found that patients' knowledge of the disease and its complications was limited. A large } \\
\text { number of patients in the case and control groups ( } 70-76 \% \text { ) were not satisfied with their knowledge. } \\
\text { This finding suggested a lack of education about LC and a lack of knowledge in both control and } \\
\text { case groups. The results of this study showed that implementing nursing intervention protocols in } \\
\text { LC patients significantly improved patient knowledge. The scores in all aspects of the Chronic Liver } \\
\text { Disease Questionnaire and the overall HRQOL score after nursing interventions were significantly } \\
\text { higher in the case group than in the control group before and after the intervention. }\end{array}$ \\
\hline Nouh et $\mathrm{al}^{15}$ & 2015 & Egypt & $\begin{array}{l}200 \text { (without } \\
\text { range of age) }\end{array}$ & $\begin{array}{l}\text { This study was performed on three groups of patients with different degrees of LC and one control } \\
\text { group. It showed a significant difference between occupation and education level of patients. In } \\
\text { addition, HRQOL was lower in patients with the advanced stage of disease. }\end{array}$ \\
\hline Tasia et $\mathrm{a}^{22}$ & 2014 & Taiwan & 49 (30-60 years) & $\begin{array}{l}\text { This cross-sectional study performed on LC patients showed a high correlation between physical } \\
\text { symptoms and psychosocial disturbances. Symptoms of physical and mental severity included } \\
\text { abdominal symptoms, fatigue, fluid retention, loss of appetite, general symptoms, decreased } \\
\text { concentration, and bleeding. Reduced concentration and general symptoms in patients were } \\
\text { associated with the severity and duration of the disease, respectively. }\end{array}$ \\
\hline Abdi et al ${ }^{13}$ & 2013 & Iran & 10 (39-54 years) & $\begin{array}{l}\text { In this qualitative study, the HRQOL of LC patients was affected by disease complications and } \\
\text { constraints, which ultimately caused physical, social, and psychological disturbances. Education } \\
\text { for these patients was also reported as being very important, helping patients overcome illness and } \\
\text { complications and enjoy their lives. The usefulness of cirrhosis patients was affected by complications } \\
\text { of illness and limitations in out-of-home activities due to medical interventions. The study found that } \\
\text { new conditions and complications from new situations could create urgent educational needs in } \\
\text { patients with cirrhosis. Educational interventions in the early stages of the disease would produce } \\
\text { better results. It was also found that a better understanding of the disease could help patients adapt to } \\
\text { new conditions. }\end{array}$ \\
\hline Goa et al ${ }^{10}$ & 2013 & China & 415 (18-80 years) & In this study, HRQOL and survival were higher in the control group than in the case group. \\
\hline $\begin{array}{l}\text { Karamat et } \\
\mathrm{al}^{17}\end{array}$ & 2013 & Iran & $\begin{array}{l}184 \text { (more than } \\
15 \text { years) }\end{array}$ & $\begin{array}{l}\text { In this study, QOL was reduced in patients with LC, cholestasis, or chronic hepatitis by fear, anxiety, } \\
\text { worry, frustration, and social problems. }\end{array}$ \\
\hline Loria et $\mathrm{al}^{5}$ & 2013 & & $\begin{array}{l}46 \text { (without range } \\
\text { of age) }\end{array}$ & $\begin{array}{l}\text { This study found that many factors affect the HRQOL of patients, including physical complications, } \\
\text { economic concerns, and imbalances in the immune system. }\end{array}$ \\
\hline $\begin{array}{l}\text { Nardelli et } \\
\mathrm{al}^{21}\end{array}$ & 2013 & & 60 (49-74 years) & $\begin{array}{l}\text { This study showed that patients with hepatic cirrhosis suffer from HRQOL disorder. Anxiety, } \\
\text { depression, and alexithymia significantly decrease HRQOL in patients with cirrhosis of the liver, and } \\
\text { they have a greater effect when the severity of the illness is higher or there is a history of hepatic } \\
\text { encephalopathy. }\end{array}$ \\
\hline Thiele et $\mathrm{al}^{18}$ & 2013 & Danish & 92 (40-70 years) & $\begin{array}{l}\text { In this study, performed on } 92 \text { LC patients, one out of five patients with hepatic cirrhosis had a } \\
\text { HRQOL lower than baseline. The severity of liver disease can predict low HRQOL and mortality } \\
\text { rates. Nutritional status and BMI were the determinant factors in the HRQOL score. }\end{array}$ \\
\hline
\end{tabular}

in a 24-hour period can diminish patients' QOL. Sleeping less than 6 hours each night is not recommended for adults and elderly people. ${ }^{1}$ Another factor is deficient immune system function. Patients who suffer from immune system malfunction are at a greater risk of life-threatening factors. Immune system dysfunction can have negative effects on the HRQOL and life expectancy of these patients. ${ }^{5}$

\subsection{Problems and Challenges of Patients With LC}

Patients with LC suffer from many problems in the physical, mental, social, economic, and familial aspects of life. One such problem is lack of self-care, which results in longer hospital stays. Therefore, evaluating nursing interventions with regards to the QOL in patients with CLD seems necessary. ${ }^{1}$ Depression, anxiety, worry, and hopelessness are some problems that can be seen at the onset.
LC patients have trouble finding a job, maintaining the ability to work, and receiving family care; they may be rejected by the community or their family. ${ }^{19}$ Complications of LC occur in the form of bleeding ${ }^{12}$ and hepatic encephalopathy, which also cause many problems for patients. There is a high risk of infection and cerebral attacks due to coagulation disorders in LC patients. ${ }^{10}$ Nausea, sleep deprivation, mental impairment, loss weight, fatigue, weakness, itching, and osteoporosis are common symptoms with LC. In such patients, glycogen stores are insufficient due to liver dysfunction, and the energy pattern in these patients after a few hours of starvation is similar to that of a healthy person after 2-3 days of hunger. This condition causes the catabolism of amino acids and ultimately leads to the atrophy of skeletal muscles; eventually, sarcopenia occurs. ${ }^{9}$ Weakness and muscle 
atrophy hinder patients at work, school, or in other social activities. Loria et al pointed out that the symptoms and complications of LC can have a significant social hazard for patients. Economic issues will also create many problems for cirrhotic patients. The physical problems of cirrhotic patients require expensive treatment, and most patients do not have enough income to cover such treatment. In the United States, the cost of cirrhosis, including medical treatments, hospital admissions, and indirect expenses such as loss of working time, is estimated to be more than US $\$ 2$ billion. Unfortunately, there are no accurate statistics on this issue in Iran. When LC progresses or another disease occurs, expenses will increase. The costs of liver transplantation and care for end-stage liver disease are very high. In addition, the complications and problems associated with LC affect their informal caregivers (family members and relatives). ${ }^{5}$

\subsection{Methods to Overcome Problems in LC Patients}

There are many ways to overcome the problems of LC. Physical symptoms, economical concerns, and emotional problems must be addressed in every meeting and examination. ${ }^{5}$ Dieting and exercise are other suggested ways to control the illness. Exercise can improve the power and size of patients' muscles. ${ }^{9}$ Dieting can cure PEM and sarcopenia in LC patients and is a good way to provide for nutritional needs and better metabolism. ${ }^{9}$ Using amino acids and supplements has a positive effect on the patients' QOL. Weight loss can increase the risk of HCC; therefore, LC patients should not lose weight. Weight gain for these patients is also important, because it can increase the risk of coronary artery problems as well as diabetes, especially when LC is advancing. ${ }^{10}$ Behaviors such as drinking, smoking, having regular follow-up to assess liver cancer and other complications, exercising, getting enough sleep, and the factors that affect sleeping should be considered. ${ }^{1}$

Supportive and palliative care can be useful in cirrhotic patients. Though different in concept, these two types of care do overlap. Palliative care, as defined by the WHO, can elevate a patient's QOL by improving his physical condition and meeting his psychological and emotional needs through family support. Five important factors in LC include (1) awareness, (2) education, (3) activity, (4) monitoring of the cirrhosis, (5) supportive and emotional care. Following the guidelines can help control the patient's signs and symptoms, postpone symptoms, and improve the patient's QOL and life expectancy. However, pursuing these instructions cannot provide sufficient and necessary information for all healthcare providers and patients, and clearly, there is a qualitative gap in this area. ${ }^{15}$

\subsection{The Basic Needs of Patients With LC}

It is important to increase the awareness and education of LC patients. LC patients do not have enough information about the illness or its process, its treatment, or its symptoms, and this can cause fear, stress, and anxiety in the. ${ }^{6,14}$ Education will enable patients to control their sickness, reduce their symptoms, and enjoy their lives. ${ }^{14}$ Education and self-care programs have positive effects on the HRQOL of patients. ${ }^{2}$ Abdi et al emphasized educating patients as soon as their disease is diagnosed, because they believe that existing stresses at the early stage of a cirrhosis diagnosis can stimulate behavioral changes and encouragement in patients. Another important fact is patients' first experiences in facing this disease. These experiences provide important and valuable information for the healthcare staff. ${ }^{14}$ In order to plan a proper careprogram, attention must be given to the needs and experiences of patients related to the disease. The most important requirements of these patients are categorized into three groups of support, ailment-related education, and information needs. Awareness of the sickness and its symptoms is very important for LC patients, because most of their stress and anxiety is related to the ailment, its expenses, and their lack of knowledge about it. The connection and bond between the healthcare team members and family members are important in problemsolving and improving the QOL of LC patients. A strong and effective relationship between the patient and her caregivers is one desirable factor in treating LC patients. Patients' satisfaction with the team of caregivers encourages them to continue their treatment process. ${ }^{14}$

\section{Conclusion}

The care and treatment of patients with LC is very hard and prolonged. They have many problems due to disease and its complications. ${ }^{20,21}$ Despite many warnings about cirrhosis, there is not enough awareness about it among the general population, caregivers, or the local and international care systems. In addition, there is a lack of strategies to prevent the burden of complications caused by CLD and LC. ${ }^{9,18,22}$ Supporting services are limited; they cannot meet the patients' needs or address their challenges and problems. These problems and complications severely affect patients' lives and decrease their HRQOL. It is necessary to adhere to several basic principles for improving the QOL in patients with LC: (1) Increasing education, awareness, and life expectancy in patients and their caregivers; (2) Using appropriate treatment protocols; (3) Providing frequent follow-ups and periodic visits; (4) Establishing proper relationships between members of the treatment team, patients, and patient families; (5) Understanding the patient's experiences during periods of illness; (6) Being aware of a patient's experiences aids in determining the appropriate treatment; and (7) Supportive family members and other caregivers.

The main sources of stress in cirrhotic patients are illness, medical costs, and a lack of awareness and education. In fact, poor physical conditions, the fear of consequences of the disease, and life-threatening conditions make a difficult situation for cirrhotic patients. Stressors, fear, and fatigue affect the patient's brain, social activities, and communication with other family members. Training and increasing awareness in cirrhotic patients can significantly 


\section{Review Highlights}

\section{What Is Already Known?}

LC is the end stage of liver disease that makes many problems and needs for patients with LC, and reduces their QOL.

\section{What This Study Adds?}

This study increases our awareness about factors affection QOL in LC patients, Problems, challenges, the basic needs of patients with LC, and methods to overcome problems in LC patients. After this study we know awareness and education are the key points in LC patients.

decrease stress, anxiety and psychological problems. Increasing awareness and education should be considered as the primary need for cirrhotic patients. All of these activities will lead to increased HRQOL and survival rates and will reduce the burden of problems and side effects of the disease for patients, their families, and community care systems. Achieving these conditions is difficult and requires the participation of patients, their healthcare providers, various sectors of society, and health systems. All care programs for cirrhotic patients should be continuous and coordinated. Statistical analyses such as meta-analysis is needed for future studies.

\section{Authors' Contributions}

All authors contributed equally to this article.

\section{Conflicts of Interest Disclosures}

The authors had no conflicts of interest.

\section{Ethical Approval}

Not applicable.

\section{References}

1. Kim HJ, Chu H, Lee S. Factors influencing on health-related quality of life in South Korean with chronic liver disease. Health Qual Life Outcomes. 2018;16(1):142. doi:10.1186/ s12955-018-0964-1.

2. Taha SH, Mohamed WY, Sayed Bukhary FA, Teleb SM, Gamal LM. Impact of a Designed Nursing Intervention Protocol on quality of life for liver cirrhosis patients in Minia University Hospital. IOSR Journal of Nursing and Health Science. 2015;4(4):7-15.

3. Hansen L, Leo MC, Chang MF, Zaman A, Naugler W, Schwartz J. Symptom distress in patients with end-stage liver disease toward the end of life. Gastroenterol Nurs. 2015;38(3):201210. doi:10.1097/sga.0000000000000108.

4. Saeedi E, Nasiri Toosi M, Salehi M, Khosravi Bizhaem S, Abolghasemi J. Effective factors for risk of death in patients with cirrhosis using competing risks model. Govaresh. 2016;21(1):27-34. [Persian].

5. Loria A, Escheik C, Gerber NL, Younossi ZM. Quality of life in cirrhosis. Curr Gastroenterol Rep. 2013;15(1):301. doi:10.1007/s11894-012-0301-5.

6. Aghdassi AA, Schneider A, Kahl M, et al. Analysis of lifestyle factors in patients with concomitant chronic pancreatitis and liver cirrhosis. Pancreatology. 2017;17(5):698-705 doi:10.1016/j.pan.2017.07.194

7. Fagerström C, Frisman GH. Living with liver cirrhosis: a vulnerable life. Gastroenterol Nurs. 2017;40(1):38-46. doi:10.1097/sga.0000000000000158.

8. Nishikawa H, Osaki Y. Liver cirrhosis: evaluation, nutritional status, and prognosis. Mediators Inflamm. 2015;2015:872152. doi:10.1155/2015/872152.

9. Sheikhian MR, Meysami Bonab S, Ahadi M, Talebi M Maasoomi E. Relationship between behavioral disorders and quality of life in patients with liver cirrhosis. Govaresh. 2014;19(1):20-25. [Persian].

10. Gao F, Gao R, Li G, Shang ZM, Hao JY. Health-related quality of life and survival in Chinese patients with chronic liver disease. Health Qual Life Outcomes. 2013;11:131. doi:10.1186/1477. 7525-11-131.

11. Souza NP, Villar LM, Garbin AJ, Rovida TA, Garbin CA Assessment of health-related quality of life and related factors in patients with chronic liver disease. Braz J Infect Dis. 2015;19(6):590-595. doi:10.1016/j.bjid.2015.08.003.

12. Volk ML, Kanwal F. Quality of Care in the Cirrhotic Patient. Clin TransIGastroenterol.2016;7:e166.doi:10.1038/ctg.2016.25.

13. Abdi F, Daryani NE, Khorvash F, Yousefi Z. Experiences of individuals with liver cirrhosis: a qualitative study. Gastroenterol Nurs. 2015;38(4):252-257. doi:10.1097/ sga.0000000000000122.

14. Low JTS, Rohde G, Pittordou K, et al. Supportive and palliative care in people with cirrhosis: international systematic review of the perspective of patients, family members and health professionals. J Hepatol. 2018;69(6):1260-1273. doi:10.1016/j. jhep.2018.08.028.

15. Nouh MAE, Badawy AM, Mohamed HM, Elkashash SA. Study of the impact of liver cirrhosis on health-related quality of Life in chronic hepatic patients. Afro Egyptian Journal of Infectious and Endemic Diseases. 2015;5(3):177-82. doi:10.21608/ aeji.2015.17827.

16. Šumskienė J, Kupčinskas L, Šumskas L. Health-related quality of life measurement in chronic liver disease patients. Medicina (Kaunas). 2015;51(4):201-208. doi:10.1016/j. medici.2015.06.006.

17. Keramat F, Mamani M, Torabian S, Aliparast Dostkohi M Assessment and Comparison of Health-Related Quality of Life in Chronic Hepatitis B and C Patients with Healthy Persons in Hamadan Province. Avicenna Journal of Clinical Medicine. 2013;20(2):151-159. [Persian].

18. Thiele M, Askgaard G, Timm HB, Hamberg O, Gluud LL. Predictors of health-related quality of life in outpatients with cirrhosis: results from a prospective cohort. Hepat Res Treat 2013;2013:479639. doi:10.1155/2013/479639.

19. Wang Q, Wang Y, Gao J, Han JT, Li Y-P. Development of a selfmanagement behaviour scale for liver cirrhosis. Int J Nurs Sci. 2015;2(1):73-79. doi:10.1016/j.ijnss.2014.10.015.

20. Hadi N, Montazeri A, Behboodi E. Assessment of health related quality of life in chronic liver disease patients. Payesh. 2010;9(2):165-72. [Persian].

21. Nardelli S, Pentassuglio I, Pasquale C, et al. Depression, anxiety and alexithymia symptoms are major determinants of health related quality of life (HRQoL) in cirrhotic patients Metab Brain Dis. 2013;28(2):239-243. doi:10.1007/s11011 012-9364-0.

22. Tsai LH, Lin CM, Chiang SC, Chen CL, Lan SJ, See LC. Symptoms and distress among patients with liver cirrhosis but without hepatocellular carcinoma in Taiwan. Gastroenterol Nurs 2014;37(1):49-59. doi:10.1097/sga.0000000000000020. 